\title{
AN UNPROVABLE RAMSEY-TYPE THEOREM
}

\author{
MARTIN LOEBL AND JAROSLAV NEŠETŘIL
}

(Communicated by Jeffrey N. Kahn)

\begin{abstract}
We present a new proof of the Paris-Harrington unprovable (in PA) version of Ramsey's theorem. This also yields a particularly short proof of the Ketonen-Solovay result on rapidly growing Ramsey functions.
\end{abstract}

\section{INTRODUCTION}

Perhaps the most famous unprovable combinatorial statement is the following form of the Finite Ramsey Theorem due to Harrington and Paris:

For every choice of positive integers $p, k, n$ there exists an integer $N$ with the following properties: If the set $[n, N]^{p}$ of

$\left(\right.$ FRT $\left.^{*}\right) \quad$ all $p$-element subsets of the set $\{n, n+1, \ldots, N\}$ is coloured by $k$ colours then there exists a homogeneous subset $Y$ such that $|Y| \geq \min Y$.

(A set $Y$ is said to be homogeneous if the set $[Y]^{p}$ is coloured by one colour only. The validity of FRT $^{*}$ for values $p, k, n$, and $N$ will be denoted in short by $N \stackrel{*}{\longrightarrow}(n)_{k}^{p}$.)

It follows from the (infinite) Ramsey theorem by a compactness argument that FRT ${ }^{*}$ is a true statement. Yet it has been proved by Paris and Harrington [11] that FRT ${ }^{*}$ cannot be proved in Peano Arithmetic PA (and, equivalently, in the Theory of Finite Sets). In fact, this presented perhaps the first mathematically interesting result independent from PA, thus complementing classical results of Gödel.

The original proofs, although short and elegant, heavily relied on techniques of mathematical logic. A breakthrough was presented by Ketonen and Solovay [5] who gave a combinatorial explanation of the unprovability of $\mathrm{FRT}^{*}$ by means of hierarchies of Fast Growing Functions. The proof of Solovay and Ketonen is natural and elegant, but long and rather technical. Here we present a simple proof of the Ketonen-Solovay theorem, which also yields perhaps the simplest proof of the unprovability of FRT* .

The main idea of the proof is that we work with ordinal-valued subsets of integers (which are called good systems) and in the main step we use a "shiftgraph" colouring.

Received by the editors February 20, 1990 and, in revised form, March 6, 1991.

1980 Mathematics Subject Classification (1985 Revision). Primary 02B10, 02D99, 05 A17. 
The paper is organized as follows: In $\S 1$ we present some definitions and easy properties of ordinals $<\varepsilon_{0}$. In $\S 2$ we prove the main result. Section 3 contains some remarks.

Modulo a few basic and well-known properties of fast growing functions the paper is self-contained.

\section{Preliminaries}

We need basic properties of (countable) ordinals and their arithmetic. Let $\varepsilon_{0}$ denote the first ordinal $\alpha$ with $\omega^{\alpha}=\alpha$. Explicitly, $\varepsilon_{0}=\sup \gamma_{n}$ where $\left.\gamma_{n}=\omega^{\omega^{\cdot{ }^{* \omega}}}\right\} n$. An ordinal $0<\alpha<\varepsilon_{0}$ has the unique representation-Cantor Normal Form-as

$$
\alpha=\omega^{\alpha_{1}} n_{1}+\cdots+\omega^{\alpha_{t}} n_{t},
$$

where $\alpha>\alpha_{1}>\alpha_{2}>\cdots>\alpha_{t}, n_{i}$ positive integers.

For $i=1, \ldots, t$ we put $S_{i}(\alpha)=\omega^{\alpha_{i}} n_{i}, s_{i}(\alpha)=n_{i}, s^{i}(\alpha)=\alpha_{i}$. If $\gamma_{h-1} \leq$ $\alpha<\gamma_{h}$ then $\alpha$ is said to have height $h(\alpha)=h$. Observe $h\left(s^{i}(\alpha)\right) \leq h(\alpha)-1$.

We also define $r(\alpha)$, the rank of $\alpha$, inductively as follows:

$$
\begin{gathered}
r(\alpha)=\alpha \quad \text { if } \alpha \text { is an integer; } \\
r(\alpha)=\max \left\{n_{1}, \ldots, n_{t}, t, r\left(\alpha_{1}\right), \ldots, r\left(\alpha_{t}\right)\right\} .
\end{gathered}
$$

The following are the principal definitions of our proof: A good couple is a pair $(\alpha, p)$ where $\alpha<\varepsilon_{0}, p$ is a positive integer, and $p>r(\alpha)+h(\alpha)$. A system of pairs $(\alpha, p)$ is said to be good if each pair $(\alpha, p)$ is a good couple. For the limit ordinal $\alpha<\varepsilon_{0}$ we define a canonical sequence $\alpha(n)$ : Let $\alpha=\omega^{\alpha_{1}} n_{1}+\cdots+\omega^{\alpha_{t}} n_{t}$ be the Cantor Normal Form of $\alpha$. If $\alpha_{t}=\alpha_{t}^{\prime}+1$ then we put $\alpha(n)=\omega^{\alpha_{1}} n_{1}+\cdots+\omega^{\alpha_{t}}\left(n_{t}-1\right)+\omega^{\alpha_{t}^{\prime}} n$. If $\alpha_{t}$ is limit then

$$
\alpha(n)=\omega^{\alpha_{1}} n_{1}+\cdots+\omega^{\alpha_{t}}\left(n_{t}-1\right)+\omega^{\alpha_{t}(n)} .
$$

In a sense $\alpha(n)$ is the fastest (natural) sequence of ordinals converging to $\alpha$.

We define the Hardy hierarchy (of fast growing functions) as follows:

$$
\begin{aligned}
H_{0}(n) & =n ; \\
H_{\alpha+1}(n) & =H_{\alpha}(n+1) ; \\
H_{\alpha}(n) & =H_{\alpha(n)}(n) \quad \text { if } \alpha \text { is a limit ordinal }<\varepsilon_{0} .
\end{aligned}
$$

The Hardy hierarchy is closely related to other hierarchies of fast growing functions (such as the Grzegorzyk hierarchy) and their importance lies in the following fundamental result (Wainer [13], see e.g., [1]):

Let $f$ be a provably total recursive function (in PA). Then there exists $\alpha<\varepsilon_{0}$ such that $f(n)<H_{\alpha}(n)$ for all sufficiently large $n$.

In this case we say briefly that $H_{\alpha}$ majorizes $f$. We close this section by establishing the existence of long (in fact, optimal) good systems. For a good couple $(\alpha, p)$ we define the pair $(\alpha, p)^{+}$as follows:

$$
\begin{aligned}
(\alpha+1, p)^{+} & =(\alpha, p+1) ; \\
(\alpha, p)^{+} & =(\alpha(p-h(\alpha)), p+1) \quad \text { for a limit } \alpha .
\end{aligned}
$$

It follows from the definition of the fundamental sequence that $r(\alpha(p-h(\alpha))) \leq \max \{r(\alpha), p-h(\alpha)\}$ and thus $(\alpha, p)^{+}$is again a good couple. 
Now fix an ordinal $\alpha$ and a positive integer $p$ such that $(\alpha, p+h(\alpha)+1)$ is a good couple. Consider the system $(\alpha, p+h(\alpha)+1),(\alpha, p+h(\alpha)+1)^{+}$, $\left((\alpha, p+h(\alpha)+1)^{+}\right)^{+}, \ldots,\left(\ldots(\alpha, p+h(\alpha)+1)^{+} \ldots\right)^{+}$, where we continue iterating the operation ()$^{+}$until the first coordinate reaches zero. Denote this good system by $L(\alpha, p)$ and by $l(\alpha, p)$ the number of its terms. It is easy to see for every $\alpha$ that $p<p^{\prime}$ implies $l(\alpha, p)<l\left(\alpha, p^{\prime}\right)$.

The following establishes the existence of long good systems.

Long Sequence Lemma. If $(\alpha, p+h(\alpha)+1)$ is a good couple then

$$
l(\alpha, p)>H_{\alpha}(p)-p .
$$

Proof. We prove $(*)$ by transfinite induction on $\alpha$. Obviously, for every natural number $n, l(n, p)$ is the length of the sequence $(n, p+2),(n-1, p+$ $3), \ldots,(0, p+n+2)$. Thus $l(n, p)=n+1>H_{n}(p)-p=n$.

In the induction step either $l(\alpha+1, p+h(\alpha+1)+1)=1+l(\alpha, p+h(\alpha)+2)>$ $1+H_{\alpha}(p+1)-p-1=H_{\alpha+1}(p)-p$; or (in a limit case) $l(\alpha, p+h(\alpha)+1)$ $=1+l(\alpha(p+1), p+h(\alpha)+2) \geq 1+l(\alpha(p+1), p+h(\alpha(p+1))+2)>1+$ $H_{\alpha(p+1)}(p+1)-p-1=H_{\alpha}(p+1)-p>H_{\alpha}(p)-p$. This proves $(*)$.

It follows that the length of good systems $L(\alpha, p)$ is not bounded by a provably total function. Particularly

$$
l\left(\gamma_{h}, p\right)>H_{\gamma_{h}}(p)-p
$$

for all sufficiently large $p$.

A good system where all ordinals have height $\leq h$ is called a good system of height $\left.\leq h . L\left(\gamma_{h-1}, p\right)\right)$ is a good system of height $\leq h$.

\section{AN UNPROVABLE THEOREM}

We prove the unprovability of FRT $^{*}$ in the following context: Let $S$ be a good system, let $Z$ be an $x$-element subset of $S$, and put $Z=\left\{\left(\beta_{1}, q_{1}\right), \ldots\right.$, $\left.\left(\beta_{x}, q_{x}\right)\right\}$. We call $Z$ a right $x$-set of $S$ if the ordinals $\beta_{1}, \ldots, \beta_{x}$ are pairwise distinct and $\beta_{i}>\beta_{j}$ iff $q_{i}<q_{j}$. A colouring of all right $x$-sets of a good system $S$ by $y$ colours is called an $(x, y)$-Paris colouring if there is not a subsystem $S^{\prime}$ of $S, S^{\prime}=\left(\beta_{1}, q_{1}\right), \ldots,\left(\beta_{m}, q_{m}\right)$ with the properties: (i) all $x$-sets in $S^{\prime}$ are right and each receives the same colour; (ii) $m \geq \min \left\{q_{1}, \ldots, q_{m}\right\}$.

Observe that all $x$-sets of a system $L\left(\gamma_{h}, p\right)$ are right $x$-sets.

The following is the key step in our proof.

Colouring Lemma. Let $S$ be a good system of height $\leq h, h \geq 2$. Assume that $h+1<q$ for all $(\alpha, q) \in S$. Then for every $y \geq 3^{(h+1)^{2}+1}$ there exists an $(h+1, y)$-Paris colouring of $S$.

Before proving the Colouring Lemma we show how it implies the unprovability of $\mathrm{FRT}^{*}$. Recall that $r^{*}(p, k, n)=\min \left\{N ; N \stackrel{*}{\longrightarrow}(n)_{k}^{p}\right\}$.

Corollary. For every $h \geq 2$

$$
r^{*}\left(h+1,3^{(h+1)^{2}+1}, 2 h+1\right) \geq H_{\gamma_{h-1}}(2 h+1)-2 h-1 .
$$

Proof. Put explicitly

$$
L\left(\gamma_{h-1}, p\right)=\left\{\left(\alpha_{1}, p_{1}\right), \ldots,\left(\alpha_{N}, p_{N}\right)\right\}, \quad p_{1}<\cdots<p_{N} .
$$


Observe that $\left(\alpha_{1}, p_{1}\right)=\left(\gamma_{h-1}, p+h+1\right)$ and $p_{i+1}=p_{i}+1$ for $i=1, \ldots, N-1$. By the Long Sequence Lemma we have $N \geq H_{\gamma_{h-1}}(p)-p$. Put $x=h+1$ and $y=3^{(h+1)^{2}+1}$. By the Colouring Lemma there exists an $(x, y)$-Paris colouring of $L\left(\gamma_{h-1}, p\right)$.

As all $x$-sets in system $L\left(\gamma_{h-1}, p\right)$ are good, this colouring induces a colouring of the set of all $x$-sets in the set $\left\{p_{1}, \ldots, N\right\}$. Thus $r^{*}\left(h+1, y, p_{1}\right)>N$. Putting $p=h$ we get

$$
r^{*}\left(h+1,3^{(h+1)^{2}+1}, 2 h+1\right) \geq H_{\gamma_{h-1}}(h)-h .
$$

Thus $r^{*}\left(h+1,3^{(h+1)^{2}+1}, 2 h+1\right)$ fails to be a provably total function.

Proof of Colouring Lemma. Let $\alpha>\beta$ be two ordinals. We put

$$
\Delta(\alpha, \beta)=\min \left\{i ; S_{i}(\alpha) \neq S_{i}(\beta)\right\} .
$$

Recall that $S_{i}(\alpha)$ is the $i$ th term in the Cantor Normal Form of $\alpha$. For a given $m$-set $\beta_{1}>\beta_{2}>\cdots>\beta_{m}$ of ordinals, $m \geq 3$, we define the shift vector $v=\left(v_{1}, \ldots, v_{m-2}\right)$ as follows:

If $\Delta\left(\beta_{i+1}, \beta_{i+2}\right)<\Delta\left(\beta_{i}, \beta_{i+1}\right)$ then $v_{i}=\nearrow$.

If $\Delta=\Delta\left(\beta_{i+1}, \beta_{i+2}\right) \geq \Delta\left(\beta_{i}, \beta_{i+1}\right)=\Delta^{\prime}$ and $s_{\Delta^{\prime}}\left(\beta_{i}\right)>s_{\Delta}\left(\beta_{i+1}\right)$ then $v_{i}=\uparrow$.

In all other cases we put $v_{i}=\downarrow$.

Example. Let $s$-set $\left(\alpha_{1}, \alpha_{2}, \alpha_{3}, \alpha_{4}, \alpha_{5}\right)$ be given as

$$
\begin{aligned}
& \alpha_{1}=\omega^{6} \cdot 4+\omega^{5}, \\
& \alpha_{2}=\omega^{6} \cdot 3+\omega^{4} \cdot 2, \\
& \alpha_{3}=\omega^{6} \cdot 3+\omega^{4}, \\
& \alpha_{4}=\omega^{6}+\omega^{4}+\omega^{2} \cdot 3, \\
& \alpha_{5}=\omega^{6}+\omega^{4}+\omega \cdot 3+5 .
\end{aligned}
$$

Then the shift vector of $\left(\alpha_{1}, \alpha_{2}, \alpha_{3}, \alpha_{4}, \alpha_{5}\right)$ is the triple $(\uparrow, \nearrow, \downarrow)$.

The definition of the coordinates of the shift vector is schematically depicted in Figure 1.

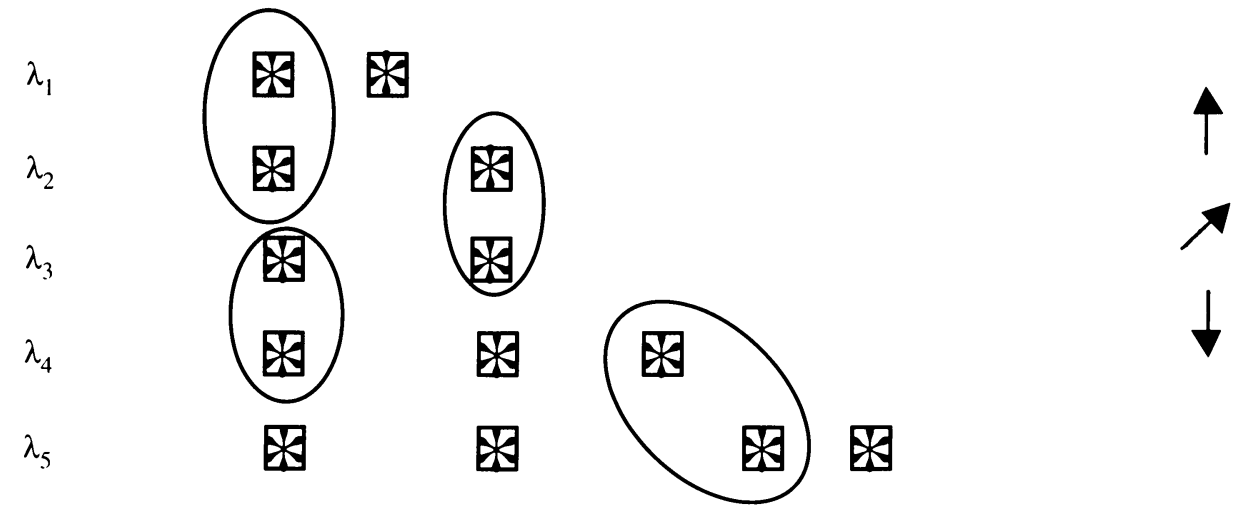

Figure 1 
We prove the Colouring Lemma by induction on $h$. For $h=2$ we assign to every triple $\left(\beta_{1}, \beta_{2}, \beta_{3}\right)$ its shift vector $\left(v_{1}\right)$ of length 1 . This is clearly a 3 -colouring. We prove that this is indeed a $(3,3)$-Paris colouring: Let $A=$ $\left(\beta_{1}, q_{1}\right), \ldots,\left(\beta_{m}, q_{m}\right)$ be a subsystem of $S$ such that each of its triple is right and monochromatic. Let this colour be $\nearrow$. Then $m \leq 1+\max \left\{t ; s_{t}\left(\beta_{1}\right) \neq\right.$ $0\}<q_{1}=\min \left\{q_{1}, \ldots, q_{m}\right\}$. If the colour is $\uparrow$ then $m \leq 1+s_{\Delta}\left(\beta_{1}\right)$ where $\Delta=\Delta\left(\beta_{1}, \beta_{2}\right)$. As $s_{\Delta}\left(\beta_{1}\right)<q_{1}$ we again have $|A|=m<\min \left\{q_{1}, \ldots, q_{m}\right\}$. If the colour is $\downarrow$ then we can use the same argument as in the case $\uparrow$ : our ordinals have height $\leq 2$ and thus have form $\omega^{a_{1}} n_{1}+\cdots+\omega^{a_{t}} n_{t}$ where $a_{1}>\cdots>a_{t}$ are integers and thus $m \leq 1+s^{\Delta}\left(\beta_{1}\right)$, where $\Delta=\Delta\left(\beta_{1}, \beta_{2}\right)$. Again $s^{\Delta}\left(\beta_{1}\right)<q_{1}$ and $|A|<\min \left\{q_{1}, \ldots, q_{m}\right\}$.

In the induction step we assume that the Colouring Lemma holds for an integer $h$ (i.e., for colouring of $h+1$ tuples) and we prove it for $h+1$. Thus let $\left(\beta_{1}, q_{1}\right), \ldots,\left(\beta_{h+2}, q_{h+2}\right)$ be a right $(h+2)$-tuple, $\beta_{1}>\cdots>\beta_{h+2}$. Let $v_{1}$ be the shift vector corresponding to $\left(\beta_{1}, q_{1}\right), \ldots,\left(\beta_{h+1}, q_{h+1}\right)$ and $v_{2}$ be the shift vector corresponding to $\left(\beta_{2}, q_{2}\right), \ldots,\left(\beta_{h+2}, q_{h+2}\right)$. We shall then assign to $\left(\beta_{1}, q_{1}\right), \ldots,\left(\beta_{h+2}, q_{h+2}\right)$ the colour $\left(v_{1}, v_{2}\right)$ with the exception of the following situation: If $v_{1}=v_{2}=(\downarrow \downarrow \downarrow \cdots \downarrow) \downarrow$ ) then the colour assigned to $\left(\beta_{1}, q_{1}\right), \ldots,\left(\beta_{h+2}, q_{h+2}\right)$ is equal to the colour of the $(h+1)$-tuple $\left(\delta_{1}, t_{1}\right), \ldots,\left(\delta_{h+1}, t_{h+1}\right)$ defined as follows: $t_{i}=q_{i}-1, \delta_{i}=s^{\Delta_{i}}\left(\beta_{i}\right)$ where $\Delta_{i}=\Delta\left(\beta_{i}, \beta_{i+1}\right)$. This colouring is guaranteed by the induction assumption as it is easy to check that all $\left(\delta_{i}, t_{i}\right)$ are good couples, thus forming a good system of height $\leq h-1$. It follows from $v_{1}=v_{2}$ that $\left(\delta_{1}, t_{1}\right), \ldots,\left(\delta_{h+1}, t_{h+1}\right)$ is a right $(h+1)$-tuple.

It remains to be proved that the constructed colouring is an $(h+2, y)$-Paris colouring. Let $A=\left(\beta_{1}, q_{1}\right), \ldots,\left(\beta_{m}, q_{m}\right)$ be a subsystem of $S$ such that each $(h+2)$-tuple is right and monochromatic. If this colour is of type $\left(v_{1}, v_{2}\right)$ and $v_{1} \neq v_{2}$ then clearly $m \leq h+2$. As $q_{i}<h+1$ for every $i=1, \ldots, m$ we have $m<\min \left\{q_{1}, \ldots, q_{m}\right\}$. Let the colour be of type $\left(v_{1}, v_{2}\right)=(v, v)$ and either $v=(\nearrow \nearrow \cdots \nearrow)$ or $v=(\uparrow \uparrow \cdots \uparrow)$. Then we conclude similarly as in the above case $h=2$ that $m \leq \min \left\{q_{1}, \ldots, q_{m}\right\}$. It remains to consider the case $v=\downarrow \downarrow \cdots \downarrow$ when the colour is defined by induction. However, then the above defined system $\left(\delta_{1}, t_{1}\right), \ldots,\left(\delta_{m-1}, t_{m-1}\right)$ has all its $(h+1)$-tuples right and monochromatic. Hence by the induction assumption $m-1 \leq t_{1}=$ $\min \left\{t_{1}, \ldots, t_{m-1}\right\}=q_{1}-1$. Thus again $m \leq \min \left\{q_{1}, \ldots, q_{m}\right\}$.

\section{CONCLUDING REMARKS}

1. There are other examples of unprovable combinatorial statements. These include a finite miniaturization of the Erdös-Rado canonization lemma (due to Kanamori-McAloon [4]), Goodstein sequences ([3], Paris-Kirby [6], for a short proof see [2]), the Hercules-Hydra game (Paris-Kirby [6]), a finite miniaturization of the Kruskal theorem (due to Friedman, see [12]), and a particular form of the Set Union Problem [9]. It seems that the Hercules-Hydra type games play a prominent role in this list as they yield particularly simple proofs of several of these results.

In fact the present proof is an outgrowth of such an approach and the first form of it was based on the Hercules-Hydra game, see Loebl [7]. 
2. The simplicity of our proof seems to be based on the fact that our colouring uses a large number of colours (namely $3^{(h+1)^{2}+1}$ ) in order to produce a colouring of a long good system. This is not the case as one can reduce the number of colours to 2 .

Corollary. $r^{*}(p, 2, n)$ fails to be a provably total function.

Proof. Consider a set $[n, N]$ and a colouring $C$ of $[n, N]^{p}$ by $y$ colours without a relatively large homogeneous set. Let $n \geq 2 p$. Put $n^{\prime}=r(p, y, 2 p)-$ the classical Ramsey number for partitions of $p$-tuples into $y$ classes. Define a colouring $C^{\prime}$ of the set $[n, N]^{2 p}$ by two colours as follows: A set $A \in[n, N]^{2 p}$ gets the colour 0 iff it is homogeneous in $C$, otherwise it gets the colour 1 . Obviously every $C^{\prime}$-homogeneous set of size $\geq n^{\prime}$ is $C$-homogeneous. This proves $r^{*}(2 p, 2, n) \geq r^{*}(p, y, n)$ for every $n>n^{\prime}$.

\section{ACKNOWLEDGMENT}

We thank the referee for remarks that improved the quality of this paper.

\section{REFERENCES}

1. W. Buchholz and S. Wainer, Provably computable functions and fast growing hierarchy, Logic and Combinatorics (S. G. Simpson, ed.) Contemp. Math., vol. 65, Amer. Math. Soc., Providence, RI, 1987, pp. 179-198.

2. E. A. Cichon, $A$ short proof of two recently discovered independence results using recursion theoretic methods, Proc. Amer. Math. Soc. 87 (1983), 704-706.

3. R. L. Goodstein, On the restricted ordinal theorem, J. Symbolic Logic 9 (1944), 33-41.

4. A. Kanamori and K. McAloon, On Gödel incompleteness and finite combinatorics, Ann. Pure Appl. Logic 33 (1987), 23-41.

5. J. Ketonen and R. Solovay, Rapidly growing Ramsey functions, Ann. of Math. (2) 113 (1981), 267-314.

6. L. Kirby and J. Paris, Accessible independence results for Peano arithmetic, Bull. London Math. Soc. 14 (1982), 285-293.

7. M. Loebl, Large functions, thesis, Charles University, Prague, 1989.

8. M. Loebl and J. Matoušek, On undecidability of the weakened Kruskal theorem, Logic and Combinatorics (S. G. Simpson, ed.), Contemp. Math., vol. 65, Amer. Math. Soc., Providence, RI, 1987, pp. 275-280.

9. M. Loebl and J. Nešetřil, Linearity and unprovability of set union problem strategies, Proc. STOC, ACM, 1988.

10. J. Paris, Statements independent of arithmetic, Mathematics of Ramsey Theory (J. Nešetřil and V. Rödl, eds.), Springer-Verlag, Berlin and New York, 1990, pp. 232-245.

11. J. Paris and L. Harrington, A mathematical incompleteness in Peano arithmetic, Handbook of Mathematical Logic (J. Barwise, ed.), North-Holland, Amsterdam, 1977, pp. 1133-1142.

12. S. G. Simpson, Nonprovability of certain combinatorial finite trees, Harvey Friedman's Research in the Foundation of Mathematics (L. Harrington, M. Morley, A. Scedrov, and S. G. Simpson, eds.), North-Holland, Amsterdam, 1985, pp. 87-117.

13. S. S. Wainer, A classification of the ordinal recursive functions, Arch. Math. Logik Grundlag. 13 (1970), 136-153.

Department of Applied Mathematics, Charles University, Malostranske Nám. 25, 11800 Prague, Czechoslovakia 